\title{
Precompetitive Consensus Building to Facilitate the Use of Digital Health Technologies to Support Parkinson Disease Drug Development through Regulatory Science
}

\author{
Diane Stephenson $^{\mathrm{a}} \quad$ Robert Alexander $^{\mathrm{b}} \quad$ Varun Aggarwal $^{\mathrm{a}} \quad$ Reham Badawy $^{\mathrm{c}}$ \\ Lisa Bain ${ }^{d}$ Roopal Bhatnagar ${ }^{a}$ Bastiaan R. Bloem ${ }^{\mathrm{e}}$ Babak Boroojerdi $^{f}$ \\ Jackson Burton ${ }^{a}$ Jesse M. Cedarbaum ${ }^{a, g}$ Josh Cosman h, u David T. Dexter ${ }^{\mathrm{i}}$ \\ Marissa Dockendorfj ${ }^{j}$ E. Ray Dorseyk Ariel V. Dowling ${ }^{b}$ Luc J. W. Evers ${ }^{\text {e }}$ \\ Katherine Fisher ${ }^{h}$ Mark Frasierl Luis Garcia-Gancedo ${ }^{m}$ Jennifer C. Goldsack ${ }^{n}$ \\ Derek Hill ${ }^{a}$ Janice Hitchcock ${ }^{a}$ Michele T. Hu ${ }^{\circ}$ Michael P. Lawton ${ }^{a}$ Susan J. Lee \\ Michael Lindemann $^{p} \quad$ Ken Marek $^{w} \quad$ Nitin Mehrotra ${ }^{j}$ Marjan J. Meinders ${ }^{\mathrm{e}}$ \\ Michael Minchik $^{a}$ Lauren Oliva $^{\mathrm{h}}$ Klaus Romero $^{\mathrm{a}}$ George Roussos $^{\mathrm{a}}{ }^{\mathrm{v}}$ \\ Robert Rubens $^{b} \quad$ Sakshi Sadar ${ }^{a}$ Joseph Scheeren ${ }^{a}$ Eiichi Sengoku ${ }^{f}$ \\ Tanya Simuni ${ }^{q} \quad G^{2}$ enn Stebbins ${ }^{r} \quad$ Kirsten I. Taylor $^{p, s}$ Beatrice Yang ${ }^{t}$ \\ Neta Zach ${ }^{b}$ on behalf of the Critical Path for Parkinson's Consortium \\ ${ }^{a}$ Critical Path Institute, Tucson, AZ, USA; b Takeda, Cambridge, MA, USA; ' University of \\ Birmingham, Birmingham, UK; ${ }^{d}$ Independent Medical Writer, Philadelphia, PA, USA; \\ e Radboud University Medical Centre, Donders Institute for Brain, Cognition and Behaviour, \\ Department of Neurology, Center of Expertise for Parkinson and Movement Disorders, \\ Nijmegen, The Netherlands; ${ }^{f}$ UCB Biopharma, Brussels, Belgium; ${ }^{9}$ Coeruleus Clinical

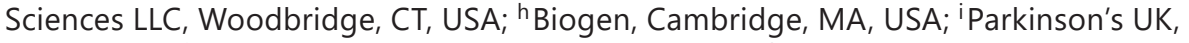 \\ London, UK; ${ }^{j}$ Merck \& Co. (MSD), Kenilworth, NJ, USA; ${ }^{~}$ University of Rochester, \\ Rochester, NY, USA; 'Michael J. Fox Foundation, New York, NY, USA; m GlaxoSmithKline, \\ Stevenage, UK; ${ }^{n}$ Digital Medicine Society, Boston, MA, USA; ${ }^{\circ}$ Nuffield Department of \\ Clinical Neurosciences, University of Oxford, Oxford, UK; ${ }^{P}$ F. Hoffmann-La Roche Ltd., \\ Basel, Switzerland; ${ }^{9}$ Northwestern University, Evanston, IL, USA; ${ }^{r}$ Rush University,

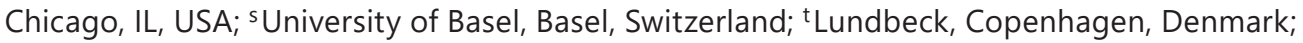

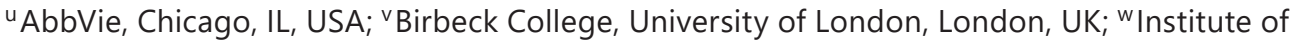 \\ Neurodegenerative Diseases, New Haven, CT, USA
}

\section{Keywords}

Digital health technologies - Regulatory science · Public-private partnerships · Collaboration · Consensus · Device agnostic

\section{Abstract}

Innovative tools are urgently needed to accelerate the evaluation and subsequent approval of novel treatments that may slow, halt, or reverse the relentless progression of Parkinson disease (PD). Therapies that intervene early in the disease continuum are a priority for the 
many candidates in the drug development pipeline. There is a paucity of sensitive and objective, yet clinically interpretable, measures that can capture meaningful aspects of the disease. This poses a major challenge for the development of new therapies and is compounded by the considerable heterogeneity in clinical manifestations across patients and the fluctuating nature of many signs and symptoms of PD. Digital health technologies (DHT), such as smartphone applications, wearable sensors, and digital diaries, have the potential to address many of these gaps by enabling the objective, remote, and frequent measurement of PD signs and symptoms in natural living environments. The current climate of the COVID-19 pandemic creates a heightened sense of urgency for effective implementation of such strategies. In order for these technologies to be adopted in drug development studies, a regulatory-aligned consensus on best practices in implementing appropriate technologies, including the collection, processing, and interpretation of digital sensor data, is required. A growing number of collaborative initiatives are being launched to identify effective ways to advance the use of DHT in PD clinical trials. The Critical Path for Parkinson's Consortium of the Critical Path Institute is highlighted as a case example where stakeholders collectively engaged regulatory agencies on the effective use of DHT in PD clinical trials. Global regulatory agencies, including the US Food and Drug Administration and the European Medicines Agency, are encouraging the efficiencies of data-driven engagements through multistakeholder consortia. To this end, we review how the advancement of DHT can be most effectively achieved by aligning knowledge, expertise, and data sharing in ways that maximize efficiencies.

(C) 2020 The Author(s)

Published by S. Karger AG, Basel

\section{Introduction}

Parkinson disease (PD) is a devastating, chronic progressive disease for which new therapies are urgently needed. The Global Burden of Disease Study reported in 2018 that PD is the fastest growing neurological disease [1]. That study estimated that in 2016 more than 6 million people worldwide had PD, i.e., more than double the number of people with PD in 1990 [1]. As the worldwide population ages, these numbers are expected to double again by about 2050 [2].

The scientific community's understanding of PD has evolved considerably since the original description of the disease 200 years ago [3]. It is now understood to be a highly heterogeneous disease characterized by both motor and nonmotor symptoms, with underlying genetic and environmental etiologies. Growing evidence suggests that long before the manifestations of classical motor signs, such as bradykinesia, rigidity, and tremor, a prodromal phase takes place that is characterized, by among others, hyposmia, sleep disturbances, depression, and constipation [4, 5]. Identifying the disease in its earliest stages is a prerequisite to slowing or stopping neurodegeneration and prevention.

The growing prevalence of PD, combined with the lack of treatments that can delay or prevent the disease or adequately address nonmotor symptoms, emphasizes the urgency of accelerated and expanded treatment options for PD across the continuum of disease, particularly in the earliest stages. Early intervention is a major focus to optimize the potential to delay or halt disease progression and it is a priority unmet need expressed by people living with PD $[6,7]$. People living with PD express significant impairments in their quality of life based on a range of both motor and nonmotor symptoms. Innovative advances in genetics and new insights into the underlying pathophysiology of the disease have contributed to a rich pipeline of therapeutics that are now in development $[8,9]$. However, PD drug development faces many challenges, including heterogeneity of signs and symptoms both across 
Digital

Biomarkers
Digit Biomark 2020;4(suppl 1):28-49

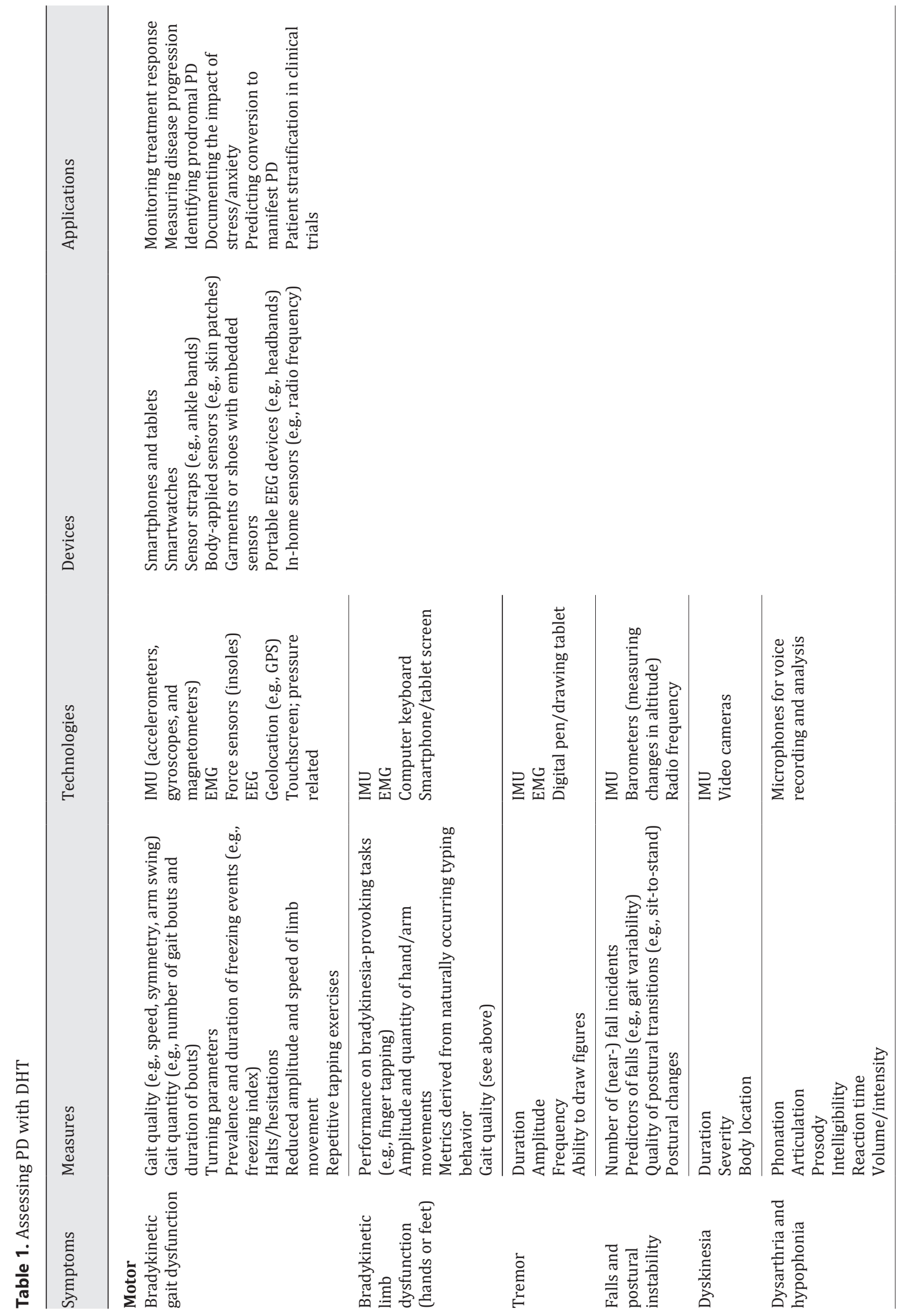


Digital

Biomarkers
Digit Biomark 2020;4(suppl 1):28-49

DOI: $10.1159 / 000512500$

(c) 2020 The Author(s).

Stephenson et al.: DHT to Enable Parkinson Clinical Trials

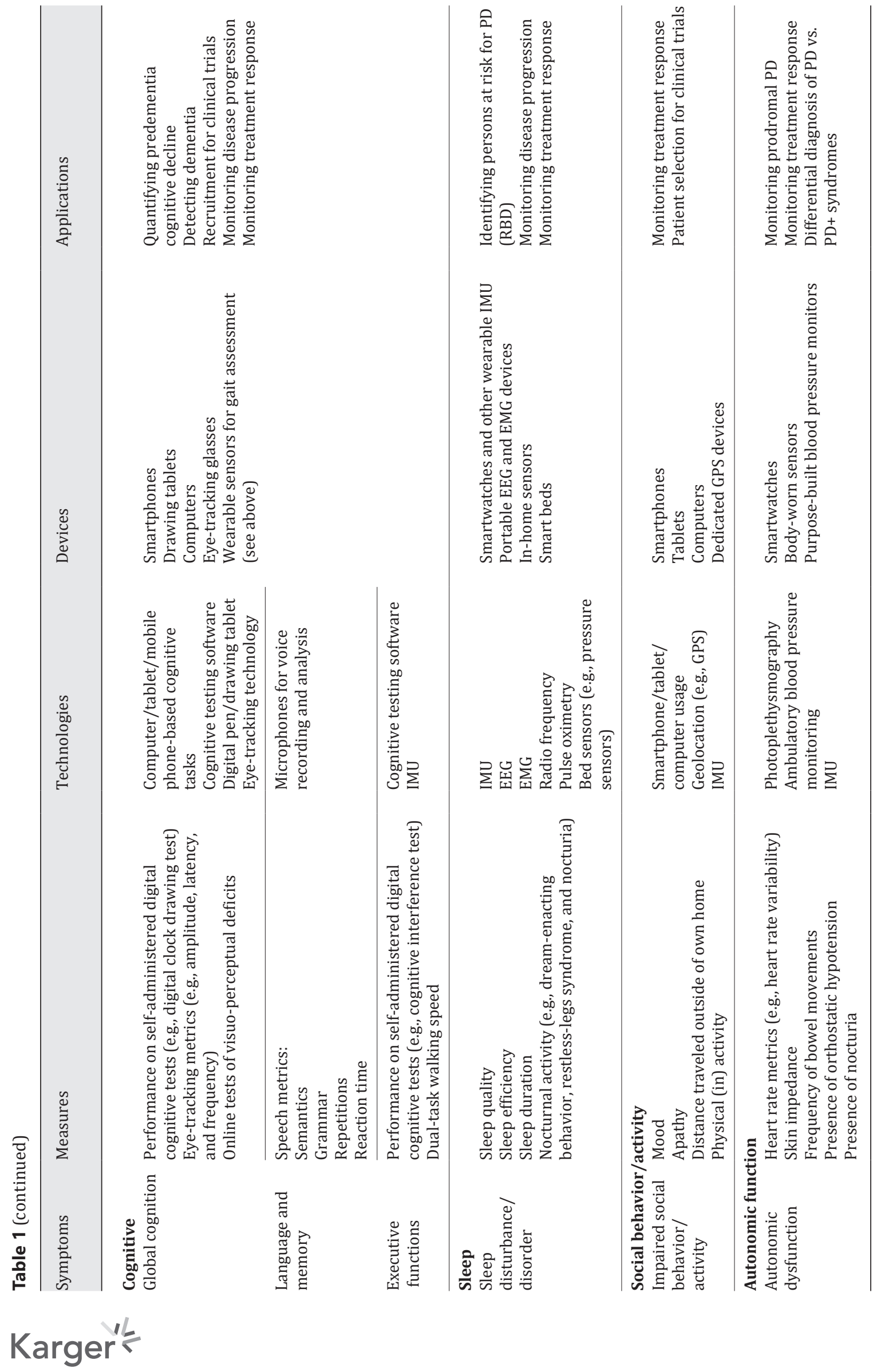


patients and - even within patients - across the disease continuum, a lack of biomarkers for patient selection and to quantify disease progression, large and heterogeneous placebo responses, and a high rate of failure of clinical trials [10]. Digital technologies are now being implemented in PD observational studies and clinical trials in both a passive and an active manner, and there is an opportunity to enable improved assessment of aspects of the disease that have been difficult to measure previously $[11,12]$.

Digital health technologies (DHT) have the potential to help address the challenges associated with developing drugs for the treatment of PD. In this review, DHT are defined as a broad range of technologies that capture information about motor presentation and mobility, pathophysiological manifestations, cognitive decline, sleep, social interactions and behaviors, activity, speech, and mood (Table 1). The terminology of DHT was selected to align with that used by regulatory agencies including the US Food and Drug Administration (FDA) and the European Medicines Agency (EMA). Categories of DHT include mobile health, health information technology, wearable devices, telehealth, and remote trials. These mobile devices have the potential to assess behavioral, cognitive, and physiological aspects of a disease in a reliable, quantifiable, objective, and continuous manner.

The effective use of DHT in clinical trials is a topic of growing interest due to their potential to improve outcome measures, aid in patient enrollment and stratification, enable monitoring of treatment adherence, enable collection of real-world data, and facilitate the implementation of remote trials [13]. However, successful deployment of DHT in PD clinical trials presents multiple challenges.

\section{Challenges and Barriers to Advancing DHT for Use in PD Trials}

As the rapidly evolving field of DHT moves forward, many challenges are emerging. The list below illustrates examples of barriers to be addressed or overcome at the present time, some which have been reviewed by others (e.g., $[14,15])$.

- Clinical relevance

- Scarcity of reliable and frequent ground truth labels in real-world conditions, making it challenging to demonstrate clinical validity

- Challenges to extract clinically meaningful information from passive and actively collected digital device data

- Data and devices

- The raw data can be complex, and meaningful signals must be filtered from a noisy background

- Lack of standardized methods for collecting, storing, organizing, curating and analyzing data

- Adapting DHT solutions to rapidly evolving aspects of the technology

- Focal placement of biomechanical sensors in defined anatomic sites requires extensive validation to establish interpretable correlations with function, disability, and ultimately quality of life

- Lack of a consensus on methods for establishing interchangeability of data obtained from different mobile devices and platforms developed by different manufacturers

- Challenges of interoperability of data as hardware and software evolve over time; lack of a consensus and success for integration of digital device studies carried out across distinct and within individual platforms

- Strategies for assessing the impact and adjusting for upgrades in hardware, software, and firmware are needed

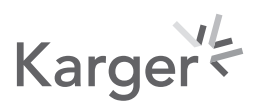


- $\quad$ Patient population, privacy, and adherence

- Issues with participant diversity, especially with regard to access and digital literacy

- Patient and caregiver engagement, especially adherence to continued use of devices over time, is modest in some cases

- Lack of alignment on best practices to ensure the utmost compliance with patient privacy

- Regulatory

- Alignment on methods to establish reliability and validity of DHT measures

- Health authority acceptance of fit-for-purpose DHT endpoints for clinical trials

The issues outlined above are difficult to address by any single individual entity. Precompetitive collaborations and public-private partnerships have been proposed as efficient means to address gaps in the development of DHT and their optimal use in drug development [16]. The opportunity to achieve a consensus among multidisciplinary stakeholders is one of the many reasons why public-private partnerships have grown in number. Integration across consortia can be an effective way to tackle challenges such as clinical meaningfulness in chronic diseases [17]. To achieve a consensus as efficiently as possible, data-driven paths that include the views of academic experts, technical, clinical, and health authority views, as well as those of nonprofit organizations and patients, need to be aligned. The objective in achieving a framework of integrated consensus building is to include all stakeholder groups working together to share costs and risks as well as minimize duplication of efforts. Such a framework is also a catalyst given the need to evaluate multiple independent data sets. Progress in the use of DHT as medical devices is advancing given the regulatory approval of specific devices such as the Parkinson's KinetiGraph [18] and BioStamp nPoint/MC10 [19]. In the current climate of the COVID-19 crisis, there will be a growing acceptance for the use of highly capable commercial devices demonstrated to be fit for purpose for an intended application [20]. Out of scope for this review is the use of DHT as medical devices for an intended use and in decision making in the course of routine clinical care. The use of DHT to collect patient selfreported information via electronic platforms (e.g., electronic patient-reported outcomes; ePRO) is also out of scope for this review.

\section{Examples of How Collaborations Can Address Gaps}

A broad range of new precompetitive collaborations that have been launched over the past several years is now serving to engage and convene diverse stakeholders with expertise in DHT with clinical, academic, and industry researchers (e.g., [14, 16, 21-24]). Many of these efforts represent true case examples illustrating how precompetitive collaborative activities are being employed efficiently to address challenges in the field. The examples that follow are a subset of multiple initiatives to highlight how collaborations can address gaps in the application of DHT with PD as the key focus; see Table 2 for a high-level summary.

\section{The Digital Medicine Society}

One notable example that crosses many disease areas is the Digital Medicine Society (DiMe), which represents the first professional organization of experts from all disciplines comprising the diverse field of digital medicine. The DiMe is a non-profit organization dedicated to advancing digital medicine to optimize human health. It serves professionals at the intersection of the global healthcare and technology communities, supporting them in developing digital medicine through interdisciplinary collaboration, research, teaching, and the promotion of best practices. Since launching in 2019, the DiMe has engaged a diversity of key stakeholders and is aggressively tackling issues that are cross cutting and leading to recommendations for the field as a whole to follow. Three examples of recent impact include: recom-

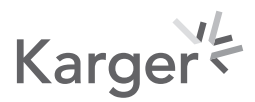


Digital

Biomarkers
Digit Biomark 2020;4(suppl 1):28-49 DOI: $10.1159 / 000512500$

(c) 2020 The Author(s). Published by S. Karger AG, Basel www.karger.com/dib

Stephenson et al.: DHT to Enable Parkinson Clinical Trials

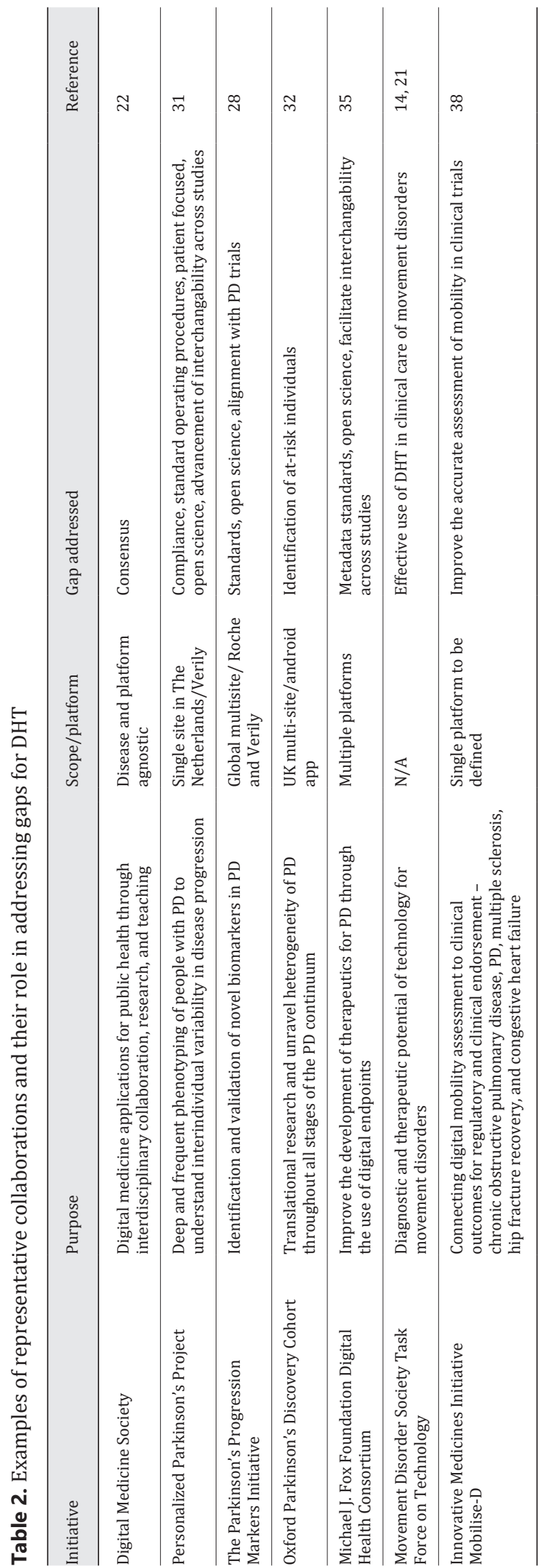


mendations for evaluation and performance of a DHT based on verification, analytical validation, and clinical validation [22]; the development of a practical guide for evaluating fit-forpurpose commercialized products across biomedical research and clinical care [25]; and the development of a framework to guide the selection and development of digital measures of health that matter to patients [26]. The DiMe has developed a crowdsourcing library of digital endpoints focused on industry-sponsored studies of new medicinal products comprised of 137 individual entries, including PD, as of October 2020 [27].

\section{Parkinson's Progression Markers Initiative}

The Parkinson's Progression Markers Initiative (PPMI) is a multicenter longitudinal study designed to comprehensively assess PD progression, clinical outcomes, imaging, digital, genetic, and biological biomarkers [28]. The study, which enrolled its first subject in 2010, is sponsored by the Michael J. Fox Foundation and it is supported in part by a precompetitive consortium that includes 29 industry and nonprofit partners. The PPMI data are openly shared as they are acquired through the PPMI website [28], with more than 5 million downloads recorded as of 2019. The impact of the PPMI continues to expand, and much of the success can be attributed to agreement on consensus data standardization and open sharing of all patient-level data with the broad community. Two distinct digital device platforms, i.e., the Verily and Roche platforms, are being utilized in PPMI subjects enrolled in USA and select non-USA sites, respectively. The Roche platform, developed by their Pharma Research and Early Development group, is a smartphone-based system that assesses PD function continuously via active monitoring of voice, balance, gait, dexterity, resting tremor, and postural tremor while participants perform a series of tasks [12,29]. Metrics under passive monitoring, such as time spent walking and sit-to-stand transitions, are collected as participants go about daily activities. The technology has been incorporated into a phase 2 clinical trial targeting $\alpha$-synuclein [12], in addition to being integrated into the PPMI multisite global observational study. The Verily platform consists of a highly advanced smartwatch developed by Verily Life Sciences. The Verily Study Watch enables the collection of physiological and environmental data about acceleration/orientation, pulse rate, electrodermal activity, electrocardiogram (ECG), barometric pressure, relative humidity, environmental temperature, and ambient light level [30]. PPMI will make digital data from both platforms available to researchers with the goal of advancing independent studies and accelerate the generation of knowledge in Parkinson therapeutic development. The PPMI platform sets a precedent for other diseases to follow to enable open science and transparency.

\section{Personalized Parkinson Project}

The Personalized Parkinson Project (PPP) is a single-site observational study being carried out with participants throughout The Netherlands [31]. The PPP study is deploying the Verily smartwatch developed by Verily Life Sciences, which has now been worn by 500 patients, 260 of whom have already worn the device for more than a year, and more than 60 patients who have already worn it for more than 2 years. The Verily study watch measures a variety of physiological and environmental parameters on a continuous basis [27]. Since the start of 2020, participants also complete weekly "virtual motor examinations" at home - a series of 7 motor tasks while wearing the smartwatch - to capture bradykinesia, tremor, gait, and posture under controlled circumstances, but still at home. All participants also complete a full neurological examination annually, during both an OFF and an ON state, while wearing the smartwatch. Participants also annually receive a complete ECG as well as a Holter ECG, while wearing the smartwatch. Importantly, the dropout rate in the entire study continues to be exceptionally low at just $1 \%$ even after 3 years into the study, and the average wear time of the watch is $22 \mathrm{~h} /$ day. The platform being employed in The Netherlands was designed to

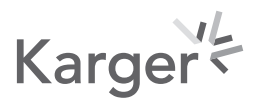


ensure a comprehensive focus on patient privacy, security, and compliance with all current regulations. An additional attribute of this study is that the PPP investigators aligned with the PPMI study investigators to enable alignment of standard operating procedures and data acquisition parameters such that that the results can be compared and integrated across studies. Opportunities to leverage this experience exist to maximize compliance in other studies. The PPP represents a unique study to begin developing digital outcome biomarkers for PD. Study data, including the raw sensor data from the smartwatch, will become available for scientists to address PD-related research questions.

\section{Oxford Parkinson's Disease Centre Discovery Cohort}

The multisite deep phenotyping study of the Oxford Parkinson's Disease Centre (OPDC) Discovery Cohort in the UK has successfully deployed smartphones for use in detecting and monitoring symptoms of PD, first in a pilot study of PD subjects and then in the largest cohort of at-risk individuals for development of PD, i.e., those with idiopathic REM sleep behavioral disorder [32]. This study is distinct in that it represents the evaluation of smartphone assessments in one of the largest cohorts of deeply phenotyped participants with idiopathic REM sleep behavioral disorder, PD, and healthy volunteers. This large cohort has only been possible due to the collaboration of multiple sites with the OPDC, and it addresses the urgent need for early detection of disease progression. The results demonstrated that objective quantification of motor symptoms using smartphones can be used to discriminate between participant groups with a high level of accuracy [32]. Postural and rest tremor were reported to be the most salient factors in discriminating idiopathic REM sleep behavioral disorder patients from controls. Successful deployment of remote monitoring technologies for identification of those who are likely to develop synucleinopathies has true potential for enabling early intervention strategies. The OPDC Discovery Cohort also used smartphones to predict the onset of clinically relevant endpoints in early PD subjects, including new-onset falls, freezing, postural instability, and cognitive and functional impairment at 18 months [33].

\section{Michael J. Fox Foundation Digital Health Consortium}

The Michael J. Fox Foundation convened a group of leading experts with the objective of improving the development of therapeutics for PD through the use of reliable digital endpoints. One gap that has been highlighted in the area of DHT is the lack of open-access consensus data standards that have been accepted by the community [34]. A diverse working group of data scientists, pharmaceutical companies, and data standard consortium partners codeveloped a comprehensive roadmap for metadata standards for DHT. This collaboration ensured that the proposed metadata set was in line with the existing needs of both research and clinical and industry stakeholders in addition to existing regulatory recommendations. Thus, the metadata standard will increase the interoperability of datasets and allow for pooling datasets in the process of validating and interpreting DHT for regulatory acceptance. The metadata set was developed specifically in the context of PD [35] as a model for a wide range of other chronic disease conditions where remote monitoring will be impactful to advance public health.

\section{Movement Disorders Society Task Force on Technology}

The Movement Disorders Society (MDS) Task Force on Technology is comprised of leading experts across different disciplines led by distinguished movement disorder experts with the goal of advancing PD research and care. The group aims to appraise the extent to which technology and data analysis can lead to actionable pharmacological and nonpharmacological therapeutics for clinical applications. A comprehensive review by the MDS task force effectively outlined gaps and opportunities [14]. A roadmap has been developed that outlines

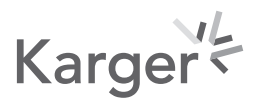


recommendations for assessing readiness for deployment of promising devices and algorithms for implementation [21]. While clinical and academic studies signify the importance of precompetitive collaborations among multidisciplinary stakeholders in addressing the existing barriers to advancing DHT for use in PD trials, alone they are not enough.

In order for precompetitive collaborations to make meaningful progress, it is important to engage with regulatory agencies early on and to implement regulatory science into the design of these studies to catalyze the use of DHT in drug trials.

\section{MDS Study Group on Telemedicine}

The MDS Study Group on Telemedicine aims to harmonize efforts to use telemedicine approaches as a way of improving care for patients with PD and other movement disorders [36]. There is considerable focus on the use of videoconferencing, in particular to support patients living in remote or otherwise underserved areas. Stimulated by the unfolding COVID-19 pandemic, this study group has also provided recommendations for the use of telemedicine to ascertain continuity of care for patients who are now grounded at home to mitigate the risk of contagion [37]. Although the focus is primarily on optimizing care delivery, many of the insights will undoubtedly prove to be useful to innovate clinical trial designs as well.

\section{Innovative Medicines Initiative Mobilise- $D$}

Mobilise-D (Connecting digital mobility assessment to clinical outcomes for regulatory and clinical endorsement; www.mobilise-d.eu) is one of the leading programs aiming to deliver a solution for remote digital assessment of mobility [38]. Mobilise-D is a public-private partnership funded by the European Innovative Medicines Initiative 2 Joint Undertaking. The Mobilise-D consortium includes 34 international research partners based at leading universities, SME, and some of the world's largest pharmaceutical and technical companies. The partners in Mobilise-D are collaborating to address the critical challenges to turn digital mobility assessment into a reality in clinical trials and ultimately healthcare. The overarching objective of Mobilise-D is to obtain regulatory approval for the use of digital mobility outcomes in a variety of disease states - i.e., PD, chronic obstructive pulmonary disease, multiple sclerosis, and proximal femoral fracture. Mobilise-D is taking an integrated approach to tackle the critical steps for adoption of digital mobility outcomes from concept, through robust technical and clinical validation, to regulatory approval. In order to achieve a lasting legacy, Mobilise-D aims to develop standards, disease and hardware agnostic approaches, open access tools, and digital databases, among other things, to harmonize working practices and aid further development. Embedded stakeholder engagement, external scientific advisory input, and patient and public involvement enrich the work of Mobilise-D to ensure maximum impact.

\section{Case Study: Engagement of Regulatory Agencies by Multidisciplinary Stakeholders to Advance DHT}

In addition to the collaborations highlighted above, the Critical Path Institute has established new initiatives under existing disease-specific consortia that are advancing the regulatory maturity of DHT.

The Critical Path Institute is an independent, nonprofit organization established in 2005 to deliver on the vision of the Critical Path Initiative of the FDA [39]. C-Path serves as a neutral convener bringing together diverse stakeholders under a legal, data-driven, and regulatory framework to streamline efficiencies in drug development across multiple disease areas [40]. The US FDA regularly engages with external public-private partners to contribute to the

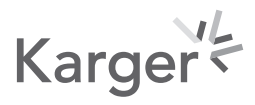


development of drug development tools, databases, consensus data standards, education/ training modules, and best practice recommendations that aim to address unmet needs [41]. C-Path also has strong alliances with the EMA and it has achieved regulatory successes across many disease areas with FDA, EMA, and PMDA.

Critical Path for Parkinson's (CPP), one of several Critical Path Institute consortia, was established as an international consortium of academic institutions, pharmaceutical companies, patient advocacy organizations, and regulators to collectively advance the development of effective treatments for PD by aligning with regulatory agencies and promoting excellence in data science using a data-driven research approach [42]. The progress of CPP to date includes qualification of dopamine transporter imaging as an enrichment biomarker for PD clinical trials in patients with early Parkinsonian symptoms [43], and the development of a global integrated unified database of standardized patient level data from clinical studies conducted around the world [42]. When CPP was launched, the concept of focusing on digital technology was envisioned as a future strategy [42]. In 2018, CPP launched a Digital Drug Development Tools (3DT) team. The goal of 3DT is to leverage the unique role of CPP as a neutral convener to bring stakeholders together in a precompetitive space to collectively engage with regulatory agencies in an iterative path to optimize the effective use of DHT in PD clinical trials.

The CPP 3DT team represents a subset of CPP member organizations (a total of 7 industry organizations as of 2020) [44]. The 3DT members are collaborating precompetitively to optimize the efficiency of paths for developing digital tools for PD drug development. This team focuses on engaging regulators early and collecting data from DHT prospectively. The nucleus of 3DT is a prospective observational study called Wearable Assessments in the Clinic and Home in PD (WATCH-PD), funded by member companies. WATCH-PD is a 12-month multicenter, longitudinal, digital assessment study of PD progression in subjects with early untreated PD (online suppl. Table 1; see www.karger.com/doi/10.1159/000512500 for all online suppl. material). The primary goal is to generate and optimize a set of candidate objective digital measures to complement standard clinical assessments in measuring the progression of disease and the response to treatment. A secondary goal is to understand the relationship between standard clinical assessments, research grade digital tools used in a clinical setting, and more user-friendly consumer digital platforms to develop a scalable approach for objective, sensitive, and frequent collection of motor and nonmotor data in early PD. The study was amended, based on the regulatory feedback described below, to include a cohort of age-matched normal subjects without PD as a parallel substudy to assess performance in normal subjects.

3DT has engaged regulators in interactive forums starting early in the project, using WATCH-PD as a pilot study around which to focus discussion. A Critical Path Innovation Meeting (CPIM) [45] on digital drug development tools for PD was held with the FDA in May 2019 on behalf of 3DT, with participating FDA staff representing 9 different offices from across the agency. CPIMs were established by the FDA to provide informal opportunities for discussing innovative drug development approaches, methodologies, and technologies. The CPIM is a means by which the FDA Center for Drug Evaluation and Research (CDER) and investigators from industry, academia, scientific consortia, patient advocacy groups, and government can communicate to improve efficiency and success in drug development. The CPIM framework represents a nonbinding platform to discuss a methodology or technology and its potential utility to enhance drug development.

Similar opportunities are available for meeting with the EMA through their Innovation Task Force (ITF) platform [46]. ITF meetings foster early dialogue on scientific, legal, and regulatory issues of emerging therapies and technologies, including digital technologies. The CPP 3DT team engaged the EMA on DHT in an ITF meeting held in July 2019.

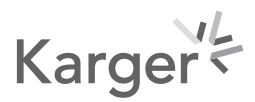


Both the FDA and the EMA expressed support for the CPP 3DT team engaging with regulators early by way of a consortium approach. The concept of collaborating early and focusing on a defined prospective pilot study was also positively perceived. The following topics were emphasized by both regulatory agencies:

- The importance of properly evaluating clinically meaningful aspects of motor, non-motor, and mood-related manifestations of PD

- The importance of assessing patients' perspective on how digital measures assess how patients function and feel

- A recommendation to conduct exit interviews to gather patient feedback on their experience with DHTs

- The importance of establishing normative databases of metrics that will be collected with wearable devices

- A suggestion that it may be beneficial to enroll subjects at the earliest point possible in disease progression to identify sensitive measures that are uniquely applicable to early $\mathrm{PD}$

- Concern about patient adherence with at-home data collection approaches and data quality

- Technical issues related to the impact of hardware/software changes on results, data quality issues, how to address missing data, and the need for transparency of algorithms

- The importance of context and accounting for environmental factors with in-home remote assessments

- Establishment of clinical meaningfulness of concepts being measured and alignment on how to assess and interpret changes in digital measures.

The EMA and FDA advised that the 3DT team proceed by adopting the recommendations and reengaging the agencies for formal review with data from multiple independent studies that will inform the appropriate target population, context of use, concept of interest, and technical validation steps needed for the future. A recommendation from the FDA was to work directly with the Office of Neuroscience of the Center of Drug Evaluation and Research and in particular with the Division of Neurology I, which regulates and reviews investigational new drug applications and marketing applications for drug and biologic products for the treatment of neurological diseases and conditions, such as Alzheimer disease, stroke, PD, Huntington disease, epilepsy, pain, muscular dystrophy, amyotrophic lateral sclerosis, multiple sclerosis, dementia, and narcolepsy.

The EMA recommendeds taking a stepwise approach, starting with the identification of small, well-defined, meaningful measures followed by a data-driven development path. When data become available, the CPP 3DT team will be poised to return to the EMA for scientific advice regarding the potential for qualification of digital measures. The EMA recommended use of the formal path defined as "qualification of novel methodologies for medicine development," a voluntary scientific pathway to evaluate novel methodologies (e.g., biomarkers, clinical outcome assessments, imaging methods, new animal models, statistical methods, innovative trial methodologies, big data approaches, etc.) to formally advance the regulatory acceptability of a specific use of a methodology for the development of medicinal products. The 3DT team has embarked on the next steps of its device-agnostic approach and is proceeding as advised with the goal of having the data inform the concepts of interest and context of use. It is anticipated that a data-driven approach and assessment of multiple independent studies will enhance alignment with healthcare regulators and effective use of DHT in PD trials.

\section{Karger'}




\section{Regulatory Science as a Catalyst to Advance the Development of DHT}

Regulatory science pathways serve to incentivize industry to align on best practices and adhere to standardized data collection and analytical approaches to ensure overall scientific rigor and clinical validity for regulatory decision making. The attention to digital technologies is featured in global regulatory agency strategic initiatives.

\section{European Medicines Agency}

The EMA clearly highlighted the importance of DHT in their "Regulatory Science to 2025" strategy document, which included the goal of exploring the application of innovative digital technologies to support data-driven decisions [47]. The EMA has issued a qualification opinion on the use of a valid and suitable wearable device to be used in Duchenne muscular dystrophy clinical trials $[48,49]$. Stride velocity 95th centile measured at the ankle was deemed an acceptable secondary endpoint in pivotal or exploratory drug therapeutic studies for regulatory purposes [50].

More recently, the EMA has outlined a framework for success in reference to deployment of DHT in clinical trials [51]. Recommendations include the following: (1) early interaction with the EMA to maximize knowledge and align across multidisciplinary teams; (2) employment of an iterative process to establish proof of concept followed by more extensive valuation of the technology; (3) identification of a clear research question; (4) attention to technologic parameters; (5) provision of appropriate documentation such as reliability, repeatability, accuracy, clinical validity, generalizability, and clinical applicability of the methodology; and (6) active encouragement and support from the EMA of advice being sought by collaborative groups such as consortia. Note that the EMA recommends that the qualification of novel methodologies serve as the platform to formally endorse the use of DHT in trials. In May 2020, the EMA issued a questions and answers guidance titled "Qualification of Digital Technology-Based Methodologies to Support Approval of Medicinal Products" that outlines concise recommendations under the Qualification of Novel Methodologies Program [52].

\section{US Food and Drug Administration}

For the FDA, there are is a growing number of examples that include the use of DHT, such as the patient-focused drug development public workshops and guidances [53], and diseasespecific guidances, such as in amyotrophic lateral sclerosis, where DHT were included for identifying and monitoring patients [54]. The concept of real-world evidence and decentralized clinical trials is advancing rapidly, with innovative regulatory science frameworks being communicated broadly [55].

As of July 2020, there were a total of 7 digital outcomes under review in the Clinical Outcome Assessment (COA) Qualification Program as digital endpoints [56].

Beyond CPP/3DT, the FDA has provided formal advice to sponsors aiming to qualify the use of DHT as digital endpoints in clinical trials. These examples span a wide range of chronic diseases, including Duchenne muscular dystrophy, multiple sclerosis, heart failure, pain, and sarcopenia. Under the 21st Century Cures Act there is transparency of materials under regulatory review for qualification of drug development tools $[57,58]$. This development enables enhanced learning opportunities for stakeholders to get a better sense of expectations for successfully advancing these tools for use in drug development. It is acknowledged that each example has unique contexts of use, yet it is likely that valuable information can be extracted to enhance the learnings and to benefit the community as a whole.

Table 3 gives insight into feedback provided by the FDA on COA qualification submissions under review that focus on DHT (passive monitoring digital COA) [56]. The column lists

\section{Karger'}


Table 3. Key issues highlighted to submitters advancing digital COA for FDA qualification

\begin{tabular}{|c|c|c|c|c|c|c|}
\hline Feedback & $\begin{array}{l}\text { Actibelt in } \\
\text { MS }^{1} \text { (No. } \\
000106)\end{array}$ & $\begin{array}{l}\text { Actibelt in } \\
\text { Sarcopenia }^{2} \\
\text { (No. } \\
\text { 000105) }\end{array}$ & $\begin{array}{l}\text { Physical Activity } \\
\text { Accelerometry } \\
\text { Assessment for } \\
\text { Analgesic Clinical } \\
\text { Trials }^{3} \\
\text { (No. 000102) }\end{array}$ & $\begin{array}{l}\text { Heart Failure } \\
\text { Activity Monitor- } \\
\text { Based Endpoint } \\
\text { Measure } \\
(\text { No. 000114) }\end{array}$ & $\begin{array}{l}\text { ActiMyo } \\
\text { in DMD } \\
\text { (No. } \\
000103 \text { ) }\end{array}$ & $\begin{array}{l}\text { Scratch Sensor } \\
\text { Atopic } \\
\text { Dermatitis } \\
\text { (No. 000120) }\end{array}$ \\
\hline Clear definition of endpoint & $\checkmark$ & $\checkmark$ & & & $\checkmark$ & \\
\hline $\begin{array}{l}\text { Patient input to determine the most important concepts and } \\
\text { aspects }\end{array}$ & $\checkmark$ & $\checkmark$ & & $\checkmark$ & $\checkmark$ & $\checkmark$ \\
\hline $\begin{array}{l}\text { Consider including those who use assistive walking devices/ } \\
\text { nonambulatory patients in the target population }\end{array}$ & $\checkmark$ & $\checkmark$ & $\checkmark$ & & & \\
\hline Test-retest to demonstrate measurement reproducibility & $\checkmark$ & $\checkmark$ & $\checkmark$ & $\checkmark$ & $\checkmark$ & \\
\hline Adequate content validity & $\checkmark$ & $\checkmark$ & $\checkmark$ & $\checkmark$ & & \\
\hline $\begin{array}{l}\text { Meaningful interpretation of variable change over time/ } \\
\text { translation of the assessed outcome into how the patient feels } \\
\text { or functions/what would be a meaningful change for patients }\end{array}$ & $\checkmark$ & $\checkmark$ & & $\checkmark$ & $\checkmark$ & \\
\hline $\begin{array}{l}\text { Effect of the sensor's weight and dimension/wearing on patient } \\
\text { movement/compliance }\end{array}$ & & & & $\checkmark$ & $\checkmark$ & \\
\hline Information on dimensions/size/weight of the sensor & $\checkmark$ & $\checkmark$ & $\checkmark$ & & & \\
\hline Description of the device, algorithm, input and output & $\checkmark$ & $\checkmark$ & $\checkmark$ & $\checkmark$ & $\checkmark$ & $\checkmark$ \\
\hline Instructions on how to use the device/details of device use & $\checkmark$ & $\checkmark$ & $\checkmark$ & & & \\
\hline $\begin{array}{l}\text { Mathematical basis of the minimum acceptable amount of } \\
\text { time for measurement/sufficient time duration for measurement }\end{array}$ & $\checkmark$ & & & & $\checkmark$ & \\
\hline $\begin{array}{l}\text { Demonstration of consistency and uniformity in measurements } \\
\text { over time in comparison to a reference value }\end{array}$ & $\checkmark$ & $\checkmark$ & & & & \\
\hline Usability testing & $\checkmark$ & $\checkmark$ & & & & $\checkmark$ \\
\hline Data loss minimization plan & $\checkmark$ & $\checkmark$ & & & & \\
\hline Plans for assessing and handling missing data & & $\checkmark$ & & $\checkmark$ & & \\
\hline $\begin{array}{l}\text { Specify patients' current activity to be referenced as baseline and } \\
\text { used for comparison after intervention }\end{array}$ & $\checkmark$ & $\checkmark$ & $\checkmark$ & & & \\
\hline Assess the effect of differences in placement of the device & $\checkmark$ & $\checkmark$ & $\checkmark$ & & & \\
\hline $\begin{array}{l}\text { Uncontrolled sources of variability should be taken into } \\
\text { consideration }\end{array}$ & $\checkmark$ & & & & & \\
\hline
\end{tabular}

Specific recommendations from the FDA for qualification submissions currently under review for application of DHT for specific contexts of use are shown. Numbers refer to the actual number assigned to individual submissions. ${ }^{1}$ Feedback ID: No. $000106 .{ }^{2}$ Feedback ID: No. $000105 .{ }^{3}$ Feedback ID: No. 000102. ${ }^{4}$ Feedback ID: No. 000114. ${ }^{5}$ Feedback ID: No. 000103. ${ }^{6}$ Feedback ID: No. 000120. More details can be found in online suppl. Table 2.

specific recommendations that were communicated to submitters as written responses to the letters of intent accepted into the qualification program.

The feedback in Table 3 was extracted from the responses to the letters of intent across each of the submissions. Additionally, more detailed feedback that is specific for that indication and context of use is not included in this table; online supplementary Table 2 provides a comprehensive list of specific feedback across the various submissions. The FDA feedback was quite consistent across the individual disease area examples. Common themes included recommendations to carry out test-retest measures for reliability, provide a complete description of the device, algorithm input and output, and outline plans for determination for meaningful interpretation of change in patients and patient input to determine important concepts and aspects for the defined target population when designing DHT studies. Separate from the FDA formal qualification pathway described above, an FDA review division pathway also may be utilized to reach alignment on acceptance for use of specific tools such as a DHT in clinical trials. 


\section{Future Directions}

The future for the advancement of digital technologies in PD is evolving in parallel with the rapid growth of the DHT landscape. The medical device and pharmaceutical industries have been working with research and clinical specialists, and patient organizations in multiple disease areas. Regulatory agencies serve a unique role to craft a framework that will guide efficient development of reliable and valid digital tools, recognizing the common challenges and opportunities faced across disease areas.

The convergence of interest in DHT from multiple stakeholder groups reflects the fact that these devices may enable the collection of clinically meaningful data from individual patients in their everyday lives. Thus, they offer opportunities to advance precision medicine. However, DHT raise substantial challenges including harmonization, standardization, validation, data storage and sharing, and privacy. Individual stakeholder groups are providing insightful recommendations for how some of these gaps can be addressed (e.g., [59, 60]). Tackling challenges collectively by advancing data-driven solutions and sharing costs and risks, as well as embracing open science, will be key to accelerating the adoption and regulatory acceptance of DHT and their use in clinical trials.

\section{Recommendations from the CPP Consortium}

The CPP outlines the following recommendations based on the collective learnings of the Critical Path Institute in leading multiple consortia across many disease areas focused on regulatory acceptance of novel drug development tools:

- Focus on what is clinically meaningful to patients as core to all aspects of PD drug development and deployment of DHT

- Build consensus on data and metadata standards that will facilitate data exchangeability and data pooling across different research and clinical trials

- Develop open-source platforms for analysis and deploy across device types and studies

- Explore opportunities to deploy remote monitoring technologies to identify individuals at risk for development of PD

- Enable data infrastructure platforms that allow the entire end-to-end workflow of digital sensor data to final measurement value

- Ensure there is transparency with provenance of data from raw data to algorithm from digital devices at all stages of the workflow; avoid black-box algorithms

- Foster paths to encourage algorithmic literacy, transparency, and oversight

- Adopt best practices as for fit-for-purpose tools aligned with recommendations by professional societies and regulators

- Adopt model-informed drug development regulatory paths by using DHT as sensitive tools to quantify disease progression

- Engage early and often with regulatory agencies via consortia that enable data sharing across multiple independent studies in a device-agnostic way

- Align directly with the review division at FDA and utilize EMA qualification of novel methodologies for medical product development

- Consider multiple applications of DHT in drug development, including monitoring of disease progression, identification of subjects at early stages of PD, dose selection in phase 1 clinical trials, and proof-of-concept decision making

- Align across relevant PD precompetitive initiatives to synergize efforts, enhance learnings, and avoid duplication of effort 


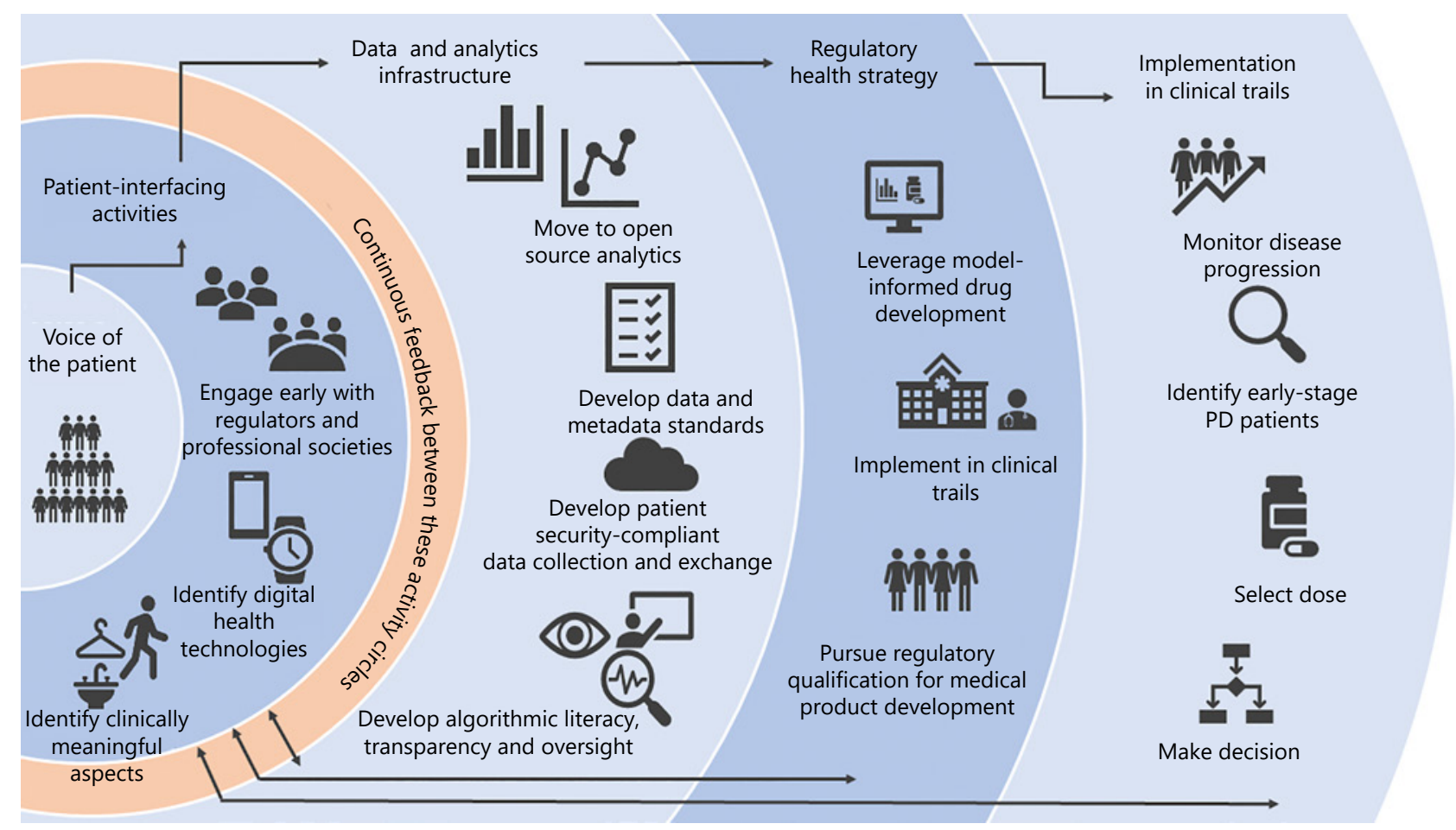

Fig. 1. Illustration of the multifaceted aspects of advancing DHT for use in PD clinical trials. Left to right: flow scheme that starts with the patient, then advances to data-driven rigor and compliance, along with regulatory acceptance and adoption in clinical trials. The original focus on the voice of the patient facilitates the identification of clinically meaningful concepts of interest. Data standardization and definition of sources of variability are key to early steps to engage regulatory agencies. Implementation in PD trials is envisioned (right) when the steps to the left are followed.

A graphical illustration of the various components of the digital technology strategy of the CPP is highlighted in Figure 1. Key components include a focus on the voice of the patient and early and frequent engagement with regulatory agencies and open science.

In summary, there are many diverse and impactful applications of how consensus building can further the effective use of DHT in advancing new treatments for PD. It is important for diverse applications to be pursued in parallel to maximize the learnings and experience across all platforms. Expertise across all stakeholders is required to address the challenges, and inclusion of regulatory agencies as part of the stakeholder community is encouraged. Alignment across efforts is needed to enhance, synergize, and minimize duplication of efforts to advance therapies that can employ DHT and provide much needed benefit for those living with PD.

\section{Acknowledgement}

The CPP acknowledges the contributions of all members of the CPP 3DT team and Parkinson's UK for their support of CPP overall. We recognize the contributions of Mark Foreman and Sreeraj Macha (representing Merck on the 3DT team). The authors acknowledge Prof. Lynn Rochester, academic lead of Mobilise-D, for her contribution. We acknowledge key CPP scientific advisors, including Ira Shoulson, Karl Kieburtz, Walter Maetzler, Camille Carroll, 
and Monica Javidnia, for their advice throughout this initiative. The CPP team recognizes the leadership of Peter Bergethon (Biogen) for initiating and supporting this project. We thank Martha Brumfield of the Critical Path Institute for her role in supporting this work from its inception. The Critical Path Institute acknowledges the Center for Human Drug Research (CHDR) in The Netherlands for engaging in informative interactions focused on validation of DHT tools. We are grateful to the following members of the US FDA for their contributions and support of CPP: Billy Dunn, Gerald Podskalny, Michelle Campbell, Chekesha Clingman, and Ameeta Parekh.

The Critical Path Institute is supported by the FDA of the US Department of Health and Human Services (HHS) and is $62 \%$ funded by the FDA/HHS totaling $\$ 14,448,917$ and $38 \%$ percent funded by non-government source(s) totaling $\$ 8,669,646$. The contents are those of the author(s) and do not necessarily represent the official views of, or an endorsement by, the FDA/HHS or the US government.

The following EMA colleagues are acknowledged for their role in supporting this initiative: Corrine de Vries and Maria Tome. The CPP graciously acknowledges the role of people living with PD in shaping this strategy.

\section{Conflict of Interest Statement}

Critical Path Institute staff (D. Stephenson, V. Aggarawal, R. Bhatnagar, K. Romero, J. Burton, S. Sadar, and M. Minchick) and R. Badawy, L. Bain, J.M. Cedarbaum, D.T. Dexter, M. Frasier, D. Hill Michael, and P. Lawton have no conflict of interests to declare. R. Alexander, N. Zach, and R. Rubens are full-time employees of Takeda. A.V. Dowling is an employee of Takeda Pharmaceuticals and also serves on the Strategic Advisory Board of the DiMe Society. B.R. Bloem currently serves as associate editor for the Journal of Parkinson Disease, serves on the editorial board of Practical Neurology and Digital Biomarkers, has received honoraria from serving on the scientific advisory board for AbbVie, Biogen, UCB, and Walk with Path, has received fees for speaking at conferences from AbbVie, Zambon, Roche, GE Healthcare, and Bial, and has received research support from The Netherlands Organisation for Scientific Research, the Michael J. Fox Foundation, UCB, AbbVie, the Stichting Parkinson-Fonds, the Hersenstichting Nederland, the Parkinson Foundation, Verily Life Sciences, Horizon 2020, Topsector Life Sciences and Health, and the Parkinson Vereniging. B. Boroojerdi and E. Sengoku are employed by UCB. J. Cosman was a full-time employee of Biogen, Inc., and is currently a full-time employee of AbbVie, Inc. M. Dockendorf, S.J. Lee, and N. Mehrotra are employees of Merck Sharp \& Dohme Corp., a subsidiary of Merck \& Co., Inc. (Kenilworth, NJ, USA), and may own stock/stock options in Merck \& Co., Inc. E.R. Dorsey has received honoraria for speaking at American Academy of Neurology courses, the American Neurological Association, and the University of Michigan. E.R. Dorsey received compensation for consulting services from: 23andMe; Abbott; AbbVie; American Well; Biogen; BrainNeuroBio; Clintrex; Curasen Therapeutics; DeciBio; Denali Therapeutics; GlaxoSmithKline; Grand Rounds; Karger; Lundbeck; MC10; MedAvante; Medical-Legal Services; Mednick Associates; the National Institute of Neurological Disorders and Stroke; the Olson Research Group; Optio; Origent Data Sciences, Inc.; Otsuka, Prilenia; Putnam Associates; Roche; Sanofi; Shire; Spark; Sunovion Pharma; Teva; Theravance; UCB; and Voyager Therapeutics. E.R. Dorsey also received research support from AbbVie, Acadia Pharmaceuticals, AMC Health, Biosensics, the Burroughs Wellcome Fund, the Davis Phinney Foundation, Duke University, the FDA, GlaxoSmithKline, the Greater Rochester Health Foundation, the Huntington Study Group, the Michael J. Fox Foundation, the National Institutes of Health/National Institute of Neurological Disorders and Stroke, the National Science Foundation, Nuredis Pharmaceuticals, the Patient-

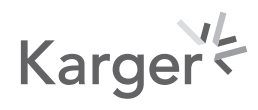


Centered Outcomes Research Institute, Pfizer, Prana Biotechnology, Raptor Pharmaceuticals, Roche, Safra Foundation, Teva Pharmaceuticals, and the University of California, Irvine; has provided editorial services for Karger Publications; and has ownership interests with Blackfynn (data integration company) and Grand Rounds (second opinion service). L.J.W. Evers has received research support from the Michael J. Fox Foundation, UCB, the Stichting Parkinson Fonds, The Netherlands Organisation for Scientific Research, and Topsector Life Sciences and Health. L. Garcia-Gancedo is a full-time employee of GSK. J.C. Goldsack is a parttime employee of HealthMode, Inc. J. Hitchcock is an employee of Hitchcock Regulatory Consulting, Inc., and has consulted for: Acumen Pharmaceuticals; Axon Advisors LLC; the Critical Path Institute; the Gerson Lehrman Group; H. Lundbeck A/S; the High Lantern Group LLC; Regenera Pharma LTD; UCB Biopharma SPRL; Vaccinex, Inc.; and Washington University. She is retired from Eli Lilly and Company and holds Lilly shares. M.T. Hu serves as a consultant for Biogen and Roche Advisory Boards and for CuraSen Therapeutics, Inc. Prof. Hu has received funding and support from Parkinson's UK, Oxford NIHR BRC, the University of Oxford, NIHR, the Michael J Fox Foundation, H2020 European Union, GE Healthcare, and the PSP Association. M. Lindemann is a consultant for F. Hoffmann-La Roche through Inovigate. M.J. Meinders has received research support from the Michael J. Fox Foundation, UCB, the Stichting Parkinson Fonds, Horizon 2020, and Topsector Life Sciences and Health. L. Oliva and K. Fisher are employees of Biogen. G. Roussos acknowledges funding from the Michael J. Fox Foundation and is a joint patent holder for UK patent GB2433856 (which is not related to healthcare). J. Scheeren was formerly employed by Bayer AG-Pharmaceuticals and as of 2019 is the president and CEO of C-Path. Dr. J. Scheeren is also currently an adjunct professor of regulatory sciences at Peking University in Beijing, China, the chair of the fellows for the Drug Information Association (DIA), a member of the advisory board for the Centre of Regulatory Excellence (CoRE), a special advisor to the scientific advisory council of the Centre for Innovation in Regulatory Science (CIRS), a lecturer at Yale University, and a foreign corresponding member of the French Académie Nationale de Pharmacie. T. Simuni, MD, has served as a consultant for Acadia, AbbVie, Accorda, Adamas, Allergan, Amneal, Aptinyx, Denali, General Electric (GE), Kyowa, Neuroderm, Neurocrine, Sanofi, Sinopia, Sunovion, Roche, Takeda, Voyager, US World Meds, the Parkinson's Foundation, and the Michael J. Fox Foundation for Parkinson research. Dr. T. Simuni has served as a speaker and received an honorarium from Acadia and Adamas, is on the scientific advisory board for Neuroderm and Sanofi, and has received research funding from the NINDS, the Parkinson's Foundation, MJFF, Biogen, Roche, Neuroderm, Sanofi, Sun Pharma, AbbVie, IMPAX, and Prevail. G. Stebbins is an employee of Rush University. Dr. G. Stebbins has consulting and advisory board membership with honoraria for: Acadia Pharmaceuticals; Adamas Pharmaceuticals, Inc.; Biogen, Inc.; Ceregene, Inc.; CHDI Management, Inc.; the Cleveland Clinic Foundation; Ingenix Pharmaceutical Services (i3 Research); MedGenesis Therapeutix, Inc.; Neurocrine Biosciences, Inc.; Pfizer, Inc.; Tools-4Patients; Ultragenyx, Inc.; and the Sunshine Care Foundation. He has received grants from and done research for: the National Institutes of Health, the Department of Defense, the Michael J. Fox Foundation for Parkinson's Research, the Dystonia Coalition, CHDI, the Cleveland Clinic Foundation, the International Parkinson and Movement Disorder Society, and CBD Solutions, and has received honoraria from: the International Parkinson and Movement Disorder Society, the American Academy of Neurology, the Michael J. Fox Foundation for Parkinson's Research, the FDA, the National Institutes of Health, and the Alzheimer's Association. K.I. Taylor is a full-time employee and shareholder of F. Hoffmann-La Roche Ltd. B. Yang is a fulltime employee at Lundbeck A/S. 


\section{Funding Sources}

CPP is funded by Parkinson's UK and the following members: AbbVie, Biogen, Cerevel, Denali, GSK, Handl Therapeutics, Lundbeck, Merck, Takeda, Sanofi, Roche, IXICO, and UCB. The 3DT project is funded by: UCB, GSK, Biogen, AbbVie, Merck, Roche, Takeda, Lundbeck, and the Michael J. Fox Foundation. We also acknowledge additional CPP member organizations, including the Parkinson's Disease Foundation, the Michael J. Fox Foundation, the Davis Phinney Foundation, the Cure Parkinson's Trust, the PMD Alliance, the University of Oxford, the University of Cambridge, Newcastle University, the University of Glasgow, and Radboud University, as well as the NINDS, the FDA, and the European Medicines Agency. Research reported in this publication was supported in part by the National Institute of Neurological Disorders and Stroke of the National Institutes of Health under award No. P50NS108676. The content is solely the responsibility of the authors and does not necessarily represent the official views of the National Institutes of Health.

\section{Author Contributions}

The corresponding author led the consortium and wrote this paper. D. Stephenson, J. Hitchcock, D. Hill, J. Cosman, and M.P. Lawton led the regulatory strategy for CPP. G. Roussos and R. Badawy are technical advisors to the 3DT project. The following authors contributed to the CPP project as members of the consortium: R. Alexander, V. Aggarawal, R. Bhatnagar, B. Boroojerdi, B.R. Bloem, J. Burton, J.M. Cedarbaum, J. Cosman, D.T. Dexter, M. Dockendorf, E.R. Dorsey, A.V. Dowling, L.J.W. Evers, K. Fisher, M. Frasier, L. Garcia-Gancedo, S.J. Lee, M. Lindemann, N. Mehrotra, M. Minchick, L. Oliva, K. Romero, R. Rubens, S. Sadar, J. Scheeren, H.E.S., G. Stebbins, K.I. Taylor, B. Yang, and N. Zach). R. Alexander and J. Cosman initiated the 3DT project under CPP and served as industry codirectors for CPP. S. Sadar and J. Burton developed Figure 1 and Tables 1-3. L. Bain contributed to Table 1 and served as scientific writer. K. Marek and T. Simuni serve as leaders of PPMI and are scientific advisors of CPP. M.T. $\mathrm{Hu}$ is academic PI of OPDC and founding member of CPP. B.R. Bloem, M. Minchick, and L.J.W. Evers are CPP members from Radboud University and PI of PPP. J.C. Goldsack is executive director of the DiMe and contributed to multiple revisions. All of the authors reviewed and approved the final submitted work.

\section{References}

1 Dorsey ER, Elbaz A, Nichols E, Abd-Allah F, Abdelalim A, Adsuar JC, et al.; GBD 2016 Parkinson's Disease Collaborators. Global, regional, and national burden of Parkinson's disease, 1990-2016: a systematic analysis for the Global Burden of Disease Study 2016. Lancet Neurol. 2018 Nov;17(11):939-53.

2 Dorsey ER, Sherer T, Okun MS, Bloem BR. The Emerging Evidence of the Parkinson Pandemic. J Parkinsons Dis. 2018;8(s1):S3-8.

3 Obeso JA, Stamelou M, Goetz CG, Poewe W, Lang AE, Weintraub D, et al. Past, present, and future of Parkinson's disease: A special essay on the 200th Anniversary of the Shaking Palsy. Mov Disord. 2017 Sep;32(9):1264310.

4 Fengler S, Liepelt-Scarfone I, Brockmann K, Schäffer E, Berg D, Kalbe E. Cognitive changes in prodromal Parkinson's disease: A review. Mov Disord. 2017 Dec;32(12):1655-66.

5 Postuma RB, Berg D, Adler CH, Bloem BR, Chan P, Deuschl G, et al. The new definition and diagnostic criteria of Parkinson's disease. Lancet Neurol. 2016 May;15(6):546-8.

6 Stephenson D. The FDA is listening: Integrating the voice of the patient in drug development for Parkinson's and Huntington's diseases [Internet]. [cited 2020 Jul 7]. Available from: https://www.mdsabstracts.org/ abstract/the-fda-is-listening-integrating-the-voice-of-the-patient-in-drug-development-for-parkinsons-andhuntingtons-diseases/. 
7 US Food and Drug Administration. The voice of the patient: Parkinson's disease [Internet]. [cited 2020 Jul 7]. Available from: https://www.fda.gov/media/124392/download.

8 Kieburtz K, Katz R, Olanow CW. New drugs for Parkinson's disease: the regulatory and clinical development pathways in the United States. Mov Disord. 2018 Jul;33(6):920-7.

9 Sardi SP, Cedarbaum JM, Brundin P. Targeted Therapies for Parkinson's Disease: From Genetics to the Clinic. Mov Disord. 2018 May;33(5):684-96.

10 Lang AE, Espay AJ. Disease Modification in Parkinson's Disease: Current Approaches, Challenges, and Future Considerations. Mov Disord. 2018 May;33(5):660-77.

11 Sim I. Mobile Devices and Health. N Engl J Med. 2019 Sep;381(10):956-68.

12 Lipsmeier F, Taylor KI, Kilchenmann T, Wolf D, Scotland A, Schjodt-Eriksen J, et al. Evaluation of smartphonebased testing to generate exploratory outcome measures in a phase 1 Parkinson's disease clinical trial. Mov Disord. 2018 Aug;33(8):1287-97.

13 Polhemus AM, Kadhim H, Barnes S, Zebrowski SE, Simmonds A, Masand SN, et al. Accelerating Adoption of Patient-Facing Technologies in Clinical Trials: A Pharmaceutical Industry Perspective on Opportunities and Challenges. Ther Innov Regul Sci. 2019 Jan;53(1):8-24.

14 Espay AJ, Bonato P, Nahab FB, Maetzler W, Dean JM, Klucken J, et al.; Movement Disorders Society Task Force on Technology. Technology in Parkinson's disease: challenges and opportunities. Mov Disord. 2016 Sep; 31(9):1272-82.

15 Pratap A, Neto EC, Snyder P, Stepnowsky C, Elhadad N, Grant D, et al. Indicators of retention in remote digital health studies: a cross-study evaluation of 100,000 participants. NPJ Digit Med. 2020 Feb;3(1):1-10.

16 Walton MK, Cappelleri JC, Byrom B, Goldsack JC, Eremenco S, Harris D, et al. Considerations for development of an evidence dossier to support the use of mobile sensor technology for clinical outcome assessments in clinical trials. Contemp Clin Trials. 2020 Apr;91:105962.

17 Ropacki MT, Hannesdottir K, Hendrix S, Gordon MF, Stephenson D, Coons SJ, et al. Clinically Meaningful Outcomes in Early Alzheimer Disease: A Consortia-Driven Approach to Identifying What Matters to Patients. Ther Innov Regul Sci. 2017 May; 51(3):380-90.

18 Farzanehfar P, Horne M. Evaluation of the Parkinson's KinetiGraph in monitoring and managing Parkinson's disease. Expert Rev Med Devices. 2017 Aug;14(8):583-91.

19 Boroojerdi B, Ghaffari R, Mahadevan N, Markowitz M, Melton K, Morey B, et al. Clinical feasibility of a wearable, conformable sensor patch to monitor motor symptoms in Parkinson's disease. Parkinsonism Relat Disord. 2019 Apr;61:70-6.

20 Bloem BR, Dorsey ER, Okun MS. The Coronavirus Disease 2019 Crisis as Catalyst for Telemedicine for Chronic Neurological Disorders. JAMA Neurol. 2020 Aug;77(8):927-8.

21 Espay AJ, Hausdorff JM, Sánchez-Ferro Á, Klucken J, Merola A, Bonato P, et al.; Movement Disorder Society Task Force on Technology. A roadmap for implementation of patient-centered digital outcome measures in Parkinson's disease obtained using mobile health technologies. Mov Disord. 2019 May;34(5):657-63.

22 Goldsack JC, Coravos A, Bakker JP, Bent B, Dowling AV, Fitzer-Attas C, et al. Verification, analytical validation, and clinical validation (V3): the foundation of determining fit-for-purpose for Biometric Monitoring Technologies (BioMeTs). NPJ Digit Med. 2020 Apr;3(1):55.

23 Byrom B, Watson C, Doll H, Coons SJ, Eremenco S, Ballinger R, et al.; ePRO Consortium. Selection of and Evidentiary Considerations for Wearable Devices and Their Measurements for Use in Regulatory Decision Making: recommendations from the ePRO Consortium. Value Health. 2018 Jun;21(6):631-9.

24 Boehme P, Hansen A, Roubenoff R, Scheeren J, Herrmann M, Mondritzki T, et al. How soon will digital endpoints become a cornerstone for future drug development? Drug Discov Today. 2019 Jan;24(1):16-9.

25 Coravos A, Khozin S, Mandl KD. Developing and adopting safe and effective digital biomarkers to improve patient outcomes. NPJ Digit Med. 2019 Mar;2(1):1-5.

26 Manta C, Patrick-Lake B, Goldsack JC. Digital Measures That Matter to Patients: A Framework to Guide the Selection and Development of Digital Measures of Health. Digit Biomark. 2020 Sep;4(3):69-77.

27 DiMe. DiMe's Crowdsourced Library of Digital Endpoints [Internet]. [cited 2020 Oct 4]. Available from: https://www.dimesociety.org/index.php/knowledge-center/library-of-digital-endpoints.

28 Marek K, Chowdhury S, Siderowf A, Lasch S, Coffey CS, Caspell-Garcia C, et al.; Parkinson's Progression Markers Initiative. The Parkinson's progression markers initiative (PPMI) - establishing a PD biomarker cohort. Ann Clin Transl Neurol. 2018 Oct;5(12):1460-77.

29 Taylor K. A comprehensive digital biomarker active testing and passive monitoring suite for the remote and frequent assessment of motor symptom progression in Parkinson's disease [Internet]. [cited 2020 Jul 7]. Available from: https://www.mdsabstracts.org/abstract/a-comprehensive-digital-biomarker-active-testingand-passive-monitoring-suite-for-the-remote-and-frequent-assessment-of-motor-symptom-progression-inparkinsons-disease/.

30 Dorsey ER, Marks WJ Jr. Verily and its approach to digital biomarkers. [Internet]. [cited 2020 Oct 4]. Available from: https://www.karger.com/Article/FullText/476051.

31 Bloem BR, Marks WJ Jr, Silva de Lima AL, Kuijf ML, van Laar T, Jacobs BP, et al. The Personalized Parkinson Project: examining disease progression through broad biomarkers in early Parkinson's disease. BMC Neurol. 2019 Jul;19(1):160.

32 Arora S, Baig F, Lo C, Barber TR, Lawton MA, Zhan A, et al. Smartphone motor testing to distinguish idiopathic REM sleep behavior disorder, controls, and PD. Neurology. 2018 Oct;91(16):e1528-38. 
33 Lo C, Arora S, Baig F, Lawton MA, El Mouden C, Barber TR, et al. Predicting motor, cognitive \& functional impairment in Parkinson's. Ann Clin Transl Neurol. 2019 Aug;6(8):1498-509.

34 Lee M, Ly H, Möller CC, Ringel MS. Innovation in Regulatory Science Is Meeting Evolution of Clinical Evidence Generation. Clin Pharmacol Ther. 2019 Apr;105(4):886-98.

35 Badawy R, Hameed F, Bataille L, Little MA, Claes K, Saria S, et al. Metadata Concepts for Advancing the Use of Digital Health Technologies in Clinical Research. Digit Biomark. 2019 Oct;3(3):116-32.

36 Ben-Pazi H, Browne P, Chan P, Cubo E, Guttman M, Hassan A, et al.; International Parkinson and Movement Disorder Society Telemedicine Task Force. The Promise of Telemedicine for Movement Disorders: an Interdisciplinary Approach. Curr Neurol Neurosci Rep. 2018 Apr;18(5):26.

37 Cubo E, Hassan A, Bloem BR, Mari Z; MDS-Telemedicine Study Group. Implementation of Telemedicine for Urgent and Ongoing Healthcare for Patients with Parkinson's Disease During the COVID-19 Pandemic: New Expectations for the Future. J Parkinsons Dis. 2020;10(3):911-3.

38 Polhemus AM, Bergquist R, Bosch de Basea M, Brittain G, Buttery SC, Chynkiamis N, et al. Walking-related digital mobility outcomes as clinical trial endpoint measures: protocol for a scoping review. BMJ Open. 2020 Jul;10(7):e038704.

39 Woodcock J, Woosley R. The FDA critical path initiative and its influence on new drug development. Annu Rev Med. 2008;59:1-12.

40 Brumfield M. The Critical Path Institute: transforming competitors into collaborators. Nat Rev Drug Discov. 2014 Nov;13(11):785-6.

41 Maxfield KE, Buckman-Garner S, Parekh A. The Role of Public-Private Partnerships in Catalyzing the Critical Path. Clin Transl Sci. 2017 Nov;10(6):431-42.

42 Stephenson D, Hu MT, Romero K, Breen K, Burn D, Ben-Shlomo Y, et al. Precompetitive Data Sharing as a Catalyst to Address Unmet Needs in Parkinson's Disease. J Parkinsons Dis. 2015;5(3):581-94.

43 Stephenson D, Hill D, Cedarbaum JM, Tome M, Vamvakas S, Romero K, et al.; Critical Path for Parkinson's Consortium. The Qualification of an Enrichment Biomarker for Clinical Trials Targeting Early Stages of Parkinson's Disease. J Parkinsons Dis. 2019;9(3):553-63.

44 Cedarbaum JM, Taylor K, Hill D, Alexander R, Luo Y, Rubens R, et al. Enabling efficient use of digital health technologies to support Parkinson's disease drug development through precompetitive collaboration [Internet]. 2019 International Congress of MDS. Available from: https://www.mdsabstracts.org/abstract/ enabling-efficient-use-of-digital-health-technologies-to-support-parkinsons-disease-drug-developmentthrough-precompetitive-collaboration/.

45 US Food and Drug Administration. Critical Path Innovation Meetings Guidance for Industry [Internet]. Available from: https://www.fda.gov/media/89497/download.

46 European Medicines Agency. Innovation in medicines [Internet]. 2018 [cited 2020 Jul 7]. Available from: https://www.ema.europa.eu/en/human-regulatory/research-development/innovation-medicines.

47 Glanville D. Regulatory science strategy [Internet]. 2018 [cited 2020 Jul 7]. Available from: https://www.ema. europa.eu/en/about-us/how-we-work/regulatory-science-strategy.

48 European Medicines Agency. Qualification opinion on stride velocity 95th centile as a secondary endpoint in Duchenne muscular dystrophy measured by a valid and suitable wearable device. Amsterdam: EMA; 2019. p. $1-77$.

49 Le Moing AG, Seferian AM, Moraux A, Annoussamy M, Dorveaux E, Gasnier E, et al. A Movement Monitor Based on Magneto-Inertial Sensors for Non-Ambulant Patients with Duchenne Muscular Dystrophy: A Pilot Study in Controlled Environment. PLoS One. 2016 Jun;11(6):e0156696.

50 Haberkamp M, Moseley J, Athanasiou D, de Andres-Trelles F, Elferink A, Rosa MM, et al. European regulators' views on a wearable-derived performance measurement of ambulation for Duchenne muscular dystrophy regulatory trials. Neuromuscul Disord. 2019 Jul;29(7):514-6.

51 Cerreta F, Ritzhaupt A, Metcalfe T, Askin S, Duarte J, Berntgen M, et al. Digital technologies for medicines: shaping a framework for success. Nat Rev Drug Discov [Internet]. 2020 May [cited 2020 Jun 29]. Available from: https://www.nature.com/articles/d41573-020-00080-6.

52 European Medicines Agency. Questions and answers: Qualification of digital technology-based methodologies to support approval of medicinal products. Amsterdam: EMA; 2020. p. 1-15.

53 US Food and Drug Administration. FDA patient-focused drug development guidance series for enhancing the incorporation of the patient's voice in medical product developmentand regulatory decision making [Internet]. FDA; 2020 [cited 2020 Jul 7]. Available from: https://www.fda.gov/drugs/development-approval-processdrugs/fda-patient-focused-drug-development-guidance-series-enhancing-incorporation-patients-voicemedical.

54 US Food and Drug Administration. Amyotrophic lateral sclerosis: Developing drugs for treatment guidance for industry [Internet]. Silver Spring: FDA; 2020 [cited 2020 Jul 7]. Available from: https://www.fda.gov/regulatory-information/search-fda-guidance-documents/amyotrophic-lateralsclerosis-developing-drugstreatment-guidance-industry.

55 Corrigan-Curay J, Sacks L, Woodcock J. Real-World Evidence and Real-World Data for Evaluating Drug Safety and Effectiveness. JAMA. 2018 Sep;320(9):867-8.

56 US Food and Drug Administration. Clinical outcome assessments (COA) qualification submissions [Internet]. Silver Spring: FDA; 2020 [cited 2020 Jul 7]. Available from: https://www.fda.gov/drugs/drug-developmenttool-ddt-qualification-programs/clinical-outcome-assessments-coa-qualification-submissions. 
57 Barlas S. The 21st Century Cures Act: FDA Implementation One Year Later: Some Action, Some Results, Some Questions. P T. 2018 Mar;43(3):149-79.

58 US Food and Drug Administration. Drug development tool qualification process: transparency provisions [Internet]. Silver Spring: FDA; 2019 [cited 2020 Jul 7]. Available from: https://www.fda.gov/drugs/drugdevelopment-tool-ddt-qualification-programs/drug-development-tool-qualification-process-transparencyprovisions.

59 Kruizinga MD, Stuurman FE, Exadaktylos V, Doll RJ, Stephenson DT, Groeneveld GJ, et al. Development of Novel, Value-Based, Digital Endpoints for Clinical Trials: A Structured Approach Toward Fit-for-Purpose Validation. Pharmacol Rev. 2020 Oct;72(4):899-909.

60 Taylor KI, Staunton H, Lipsmeier F, Nobbs D, Lindemann M. Outcome measures based on digital health technology sensor data: data- and patient-centric approaches. NPJ Digit Med. 2020 Jul;3(1):97. 